\title{
Pneumocystis jirovecii colonization and its association with pulmonary diseases: a multicenter study based on a modified loop-mediated isothermal amplification assay
}

Ting Xue ${ }^{1,2}$, Zhuang Ma ${ }^{3}$, Fan Liư ${ }^{4}$, Weiqin Dư ${ }^{5}$ Li He ${ }^{1}$, Jinyan Wang ${ }^{6}$ and Chunli An ${ }^{1 *}$

\begin{abstract}
Background: Pneumocystis jirovecii (P. jirovecii) is an opportunistic fungal pathogen and the role of its colonization in pulmonary diseases has become a popular focus in recent years. The aim of this study was to develop a modified loop-mediated isothermal amplification (LAMP) assay for detection of Pneumocystis jirovecii (P. jirovecii) DNA amongst non-HIV patients with various pulmonary diseases and use it to examine the prevalence and assess the association of $P$. jirovecii colonization with clinical characteristics of these diseases.

Methods: We modified the previously reported LAMP assay for $P$. jirovecii by adding real-time detection. This method was used to detect $P$. jirovecii colonization in pulmonary samples collected from 403 non-HIV patients with various pulmonary diseases enrolled from 5 hospitals in China. We determined the prevalence of $P$. jirovecii colonization in 7 types of pulmonary diseases and assessed the association of $P$. jirovecii colonization with clinical characteristics of these diseases.

Results: The modified LAMP assay showed no cross-reactivity with other common pulmonary microbes and was 1000 times more sensitive than that of conventional PCR. Using the modified LAMP assay, we detected P. jirovecii colonization in 281 (69.7\%) of the 403 patients enrolled. P. jirovecii colonization was more common in interstitial lung diseases than in chronic obstructive pulmonary disease (COPD) $(84.6 \%$ vs $64.5 \%, P<0.05)$. Patients with acute exacerbation of COPD had a higher prevalence of $P$. jirovecii colonization compared to patients with stabilized COPD $(67.4 \%$ vs $43.3 \%, P<0.05)$. P. jirovecii colonization was associated with decreased pulmonary function, increased levels of 1,3- $\beta$-D-glucan and $C$-reactive protein, and decreased levels of CD4+ T-cell counts $(P<0.05$ for each). Approximately $70 \%$ of $P$. jirovecii colonized patients had confections with other fungi or bacteria.

\footnotetext{
* Correspondence: cmucl@126.com

'Department of Microbiology and Parasitology, College of Basic Medical

Science, China Medical University, 77 Puhe Road, Shenyang, People's

Republic of China

Full list of author information is available at the end of the article
}

(c) The Author(s). 2020 Open Access This article is licensed under a Creative Commons Attribution 4.0 International License, which permits use, sharing, adaptation, distribution and reproduction in any medium or format, as long as you give appropriate credit to the original author(s) and the source, provide a link to the Creative Commons licence, and indicate if changes were made. The images or other third party material in this article are included in the article's Creative Commons licence, unless indicated otherwise in a credit line to the material. If material is not included in the article's Creative Commons licence and your intended use is not permitted by statutory regulation or exceeds the permitted use, you will need to obtain permission directly from the copyright holder. To view a copy of this licence, visit http://creativecommons.org/licenses/by/4.0/. The Creative Commons Public Domain Dedication waiver (http://creativecommons.org/publicdomain/zero/1.0/) applies to the data made available in this article, unless otherwise stated in a credit line to the data. 
(Continued from previous page)

Conclusions: We developed a modified LAMP assay for detecting P. jirovecii. Our multi-center study of 403 patients supports that $P$. jirovecii colonization is a risk factor for the development of pulmonary diseases and highlights the need to further study the pathogenesis and transmission of $P$. jirovecii colonization in pulmonary diseases.

Keywords: P. jirovecii, Colonization, Respiratory diseases, Loop-mediated isothermal amplification

\section{Background}

Pneumocystis jirovecii (P. jirovecii) is a unicellular parasitic fungus and an opportunistic pathogen which almost exclusively infects the lungs $[1,2]$. $P$. jirovecii infection may lead to a fatal $P$. jirovecii pneumonia (PJP) in immunocompromised patients, particularly in those infected with human immunodeficiency virus (HIV). While, the incidence of PJP in HIV-infected patients has decreased dramatically, mainly due to the widespread use of combination antiretroviral therapy, there are growing reports of PJP cases amongst HIV-negative individuals [3-7]. In addition, asymptomatic P. jirovecii colonization is being increasingly identified in immunocompetent individuals $[2,8]$. In recent years, the role of $P$. jirovecii colonization in pulmonary diseases has become a popular focus, especially in those with chronic obstructive pulmonary disease (COPD) and sudden infant death syndrome [5, 8-11]. These asymptomatic PJP individuals, carrying $P$. jirovecii, may act as an important reservoirs of nosocomial infections. Therefore, it is necessary to clarify the role of $P$. jirovecii colonization in the pathogenesis of pulmonary diseases.

Various molecular methods for detection of $P$. jirovecii have been described previously, including conventional polymerase chain reaction (PCR) and real-time quantitative PCR [12-15]. Loop-mediated isothermal amplification (LAMP), originally developed by Notomi $\mathrm{T}$ et al. [16], is a novel, highly sensitive, and specific method to diagnose infectious diseases. However, SYBR Green I, used as a conventional intercalating fluorescent dye, resulted in a certain degree of inhibition of LAMP reaction, leading to several errors in the diagnosis $[17,18]$. Thus, the aims of this study were to develop a modified real-time fluorescence LAMP assay by adding real time, and SYTO 9.0, as the fluorescent dye, for P. jirovecii detection, in order to avoid inhibition for LAMP reaction by SYBR Green I, and then, use it to examine the prevalence of $P$. jirovecii colonization amongst the non-HIV patients with various pulmonary diseases, and assess the association of $P$. jirovecii colonization with clinical characteristics of these diseases.

\section{Materials and methods Patient enrollment}

This retrospective study was approved by the Institutional Ethics Review Board, China Medical University (2013070).
Written informed consent was obtained from all the patients involved in this study. We enrolled a total of 403 patients with various pulmonary diseases hospitalized between October 2016 and January 2018, from 5 hospitals in China, including the First Affiliated Hospital of China Medical University, the Fourth Affiliated Hospital of China Medical University, the Fifth Affiliated Hospital of China Medical University, the Eleventh Affiliated Hospital of Shanxi Medical University, and the General Hospital of Shenyang Military Command. Clinical background information of patients was collected through the Case File System, available in the participating hospitals.

The patients with different symptoms and signs of pulmonary diseases, with non-HIV infections, without the clinical evidence or history of PJP symptoms, and without the definite diagnosis of PJP were included in the study $[14,19]$. Besides considering the predisposing factors for the diseases, the pulmonary diseases were diagnosed not only on the basis of the clinical manifestations of the respiratory tract diseases (such as cough, fever, expectoration, wheezing, chest pain, chest tightness, hemoptysis, and dyspnea), but also chest imaging tests, bronchoscopic examination, and laboratory test, primarily including COPD [including the stable stage and acute exacerbations (AECOPD)], acute exacerbations of chronic bronchitis (AECB), interstitial lung diseases (ILDs), bronchiectasis, bronchial asthma, invasive pulmonary aspergillosis (IPA), and type I respiratory failure. Various diseases, included in the study, were diagnosed as follows: i) COPD was diagnosed mainly by confirming the presence of persistent airflow limitation according to a post-bronchodilator FEV1/ FVC $<70 \%$, history of risk factors such as exposure to cigarette smoke, and the Global Strategy for the Diagnosis, Management, and Prevention of Chronic Obstructive Lung Disease 2017 Report: GOLD Executive Summary [20]. In addition, the assessment of AECOPD met the GOLD guidelines, the diagnostic criteria of AECOPD given by American College of Chest Physicians (2011), and the European Respiratory Society guidelines [21]; ii) ILDs were primarily diagnosed by high-resolution computed tomography (HRCT) depicting the radiological manifestations of fibrosis in ILDs and sometimes, by bronchoalveolar lavage for the diagnosis of some particular types of ILDs [22, 23]; iii) AECB was diagnosed by considering the patients with mild to moderate syndromes of COPD (the diagnosis of COPD as mentioned above), 
patients who developed the exacerbated clinical presentations with increased dyspnea, increased sputum volume, and increased sputum purulence, and meanwhile, excluding pneumonia and other lung diseases by chest radiography [24]; iv) bronchiectasis was primarily diagnosed by HRCT of the chest [25], the gold standard for confirming the bronchiectasis, and considering the underlying causes such as post-infectious [26]; v) bronchial asthma was diagnosed through a detailed clinical history, physical examination, lung function, and detection of allergens [27]; vi) IPA was diagnosed by demonstrating Aspergillus in the respiratory samples and other contributions by $1,3-\beta$-D-glucan assay, clinical manifestation of lower respiratory tract infection, and CT clinical characteristic with the aircrescent sign, halo sign, cavitation lesion, or nodules infarction; and vii) type I respiratory failure was diagnosed by laboratory studies such as evaluating the level of hypoxemia and hypercapnia via arterial blood gas and oxygen saturation, imaging examination, and pulmonary function test [28].

\section{Respiratory tract specimens}

Before patients received diagnosis, antibiotics, and other treatments, specimens including blood, bronchoalveolar lavage fluid (BALF), and sputum samples were collected. Venous blood was collected in vacutainer tubes with different anticoagulants or coagulants and used immediately for the following tests: CD4+ T-cell counts (BD FACS Canto, USA), erythrocyte sedimentation rate (ESR) (Vital Monitor-20, Italy), and serum biochemical parameters including lactate dehydrogenase (LDH) (ADVIA 2400, SIEMENS Healthineers, Germany), pro-calcitonin (PCT) (Cobas 8000, Roche Diagnostics GmbH, Germany), Creactive protein (CRP) (BN ProSpec System, SIEMENS Healthineers, Germany), and 1,3- $\beta$-D-glucan (BDG) (Goldstream MB-80, Era Biology Technology Co. Ltd. Tianjin, China). Pulmonary function (Medisoft S.A. Hyp' Air, Belgium) and high-resolution computed tomography (HRCT) (BN ProSpec System, SIEMENS Healthineers, Germany) were measured by the following standard protocols in clinical practice. Each patient underwent HIV-1 and 2 antibody testing using the anti-HIV-1 and 2 antibody enzyme-linked immunosorbent assay (ELISA) diagnostic kit (Intec Products, Inc. Xiamen, China) to determine HIV infection status.

Amongst 403 patients, 324 patients provided sputum samples and 79 patients provided BALF samples. As the nature of the study is retrospective and severity status of the pulmonary disease was not available in the Case File System, we can only assume that patients who provided BALF sample had more severe disease status, as compared to patients who provide sputum samples. Moreover, patients providing sputum samples were not eligible for the BAL procedure. To collect the sputum samples, patients gargled with saline solution approximately three times to prevent oral microbial contamination before coughing up their morning sputa from the deep respiratory tract. Sputa were then collected in sterile containers and sent to clinical laboratories for bacterial and/or fungal culture and identification, respectively using the VITEC-2 system (ATB system, BioMérieux, Marcy-l'Étoile, France) and the Sensititre ${ }^{\mathrm{Tu}}$, Aris 2X system (Thermo Fisher Scientific, USA). The remaining of the sputum samples were used to exact DNA and make slide smear to identify $P$. jirovecii microscopically following GMS and Giemsa staining.

Bronchoscopic examination was performed in 79 patients. BALF was collected using strict aseptic technique and filtered through a 2-layered nylon gauze to remove the mucus. The filtered BALF was divided for DNA extraction (described below) and slide smear staining by GMS and Giemsa staining.

\section{DNA extraction}

BALF samples were centrifuged at $1500 \mathrm{rpm}$ for $15 \mathrm{~min}$ and cell pellets were collected. The sputa were pretreated with $4 \% \mathrm{NaOH}(\mathrm{w} / \mathrm{v} \%)$, washed with saline solution approximately three times, and then centrifuged at $12,000 \mathrm{rpm}$ for $10 \mathrm{~min}$. After centrifugation, the supernatant was discarded. All cell pellets were washed three times with PBS and then subjected to DNA extraction following the traditional protocol involving proteinase $\mathrm{K}$ digestion, phenol-chloroform extraction, and ethanol precipitation. The DNA extracts were quantitated using the NanoDrop UV-Vis spectrophotometer (Thermo Fisher Scientific, USA) and stored at $-80^{\circ} \mathrm{C}$ before use.

\section{Real-time fluorescence LAMP assay}

All primers used in this study, including the external primer set (F3-B3), internal primer set (FIP-BIP), and loop primer set (LF-LB) have been described previously [5]. The LAMP reaction mixture contained $1.6 \mu \mathrm{M}$ FIP-BIP, $0.2 \mu \mathrm{M}$ F3-B3, $0.4 \mu \mathrm{M}$ Loop F-B, and $2.5 \mu \mathrm{L} 10 \times$ Isothermal Amplification Buffer including 0.1\% Triton X-100 or $100 \mu \mathrm{g} / \mathrm{ml} \mathrm{BSA}$. The preferred buffer was $50 \mathrm{mM} \mathrm{KCl}$, $10 \mathrm{mM}$ Tris- $\mathrm{HCl}$ (pH 7.5), 0.1 mM EDTA, $1 \mathrm{mM}$ DTT, $0.1 \%$ Triton X-100, and 50\% glycerol (New England Biolabs, USA), $3.5 \mu \mathrm{L} 10 \mathrm{mM}$ dNTPs (Takara Biotechnology Co., LTD., Dalian, China), $1 \mu \mathrm{l} 8 \mathrm{U} / \mu \mathrm{l}$ Bst DNA Polymerase, Large Fragment (New England Biolabs, USA), $1 \mu \mathrm{L} 100 \mathrm{mM} \quad \mathrm{MgSO}_{4}$ (New England Biolabs, USA), $0.5 \mu \mathrm{L} 10 \mu \mathrm{M}$ SYTO 9.0 (Thermo Fisher Scientific, USA), $2 \mu \mathrm{L} 5.0 \mathrm{M}$ Betaine (Sigma-Aldrich, USA), $2 \mu \mathrm{L}$ DNA template solution, and $6.5 \mu \mathrm{L}$ RNase-free water (Takara Biotechnology Co., LTD., Dalian, China) up to a final volume of $25 \mu \mathrm{L}$. Each experiment included a no template control (with distilled water). The LAMP reaction was performed in the ABI 7500 system 
(ThermoFisher Scientific, USA) at $63{ }^{\circ} \mathrm{C}$ for $60 \mathrm{~min}$, and terminated at $80^{\circ} \mathrm{C}$ for $10 \mathrm{~min}$.

The entire LAMP amplification process was monitored in real time and the fluorescent signals recorded automatically by the ABI 7500 system. Compared with the conventional LAMP, the real-time LAMP was analyzed by the amplification curves. At the end of the reaction, white precipitates formed at the bottom of the test tube. In the analysis of conventional LAMP, visual detection was observed by adding $2 \mu \mathrm{L} 1000 \times$ SYBR Green I (Thermo Fisher Scientific, USA) and the detection of LAMP amplicons were evaluated by $2 \%$ agarose gel electrophoresis and stained with GoodView (SBS Genetech Co., Ltd.) for visualization under UV radiation.

\section{Conventional PCR}

PCR was performed using the external primers targeting the 18S rRNA gene of $P$. jirovecii described elsewhere [5]. PCR mixture contained $12.5 \mu \mathrm{L}$ of DNA polymerase

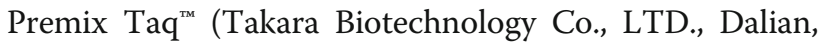
China), $1-3 \mu \mathrm{L}$ of DNA samples, $1 \mu \mathrm{L}$ of $10 \mu \mathrm{M}$ F3-B3 primer pair, and RNase-free water up to a final volume of $25 \mu \mathrm{L}$. Ultrapure distilled water was used as a negative control. PCR was performed under the following conditions: $94{ }^{\circ} \mathrm{C}$ for $5 \mathrm{~min}$; 30 cycles of $94{ }^{\circ} \mathrm{C}$ for $30 \mathrm{~s}, 55{ }^{\circ} \mathrm{C}$ for $30 \mathrm{~s}, 72{ }^{\circ} \mathrm{C}$ for $30 \mathrm{~s}$, and $72{ }^{\circ} \mathrm{C}$ for $8 \mathrm{~min}$. PCR products were separated by electrophoresis on $1.5 \%$ agarose gels, stained with GoodView (SBS Genetech Co., LTD. Beijing, China), and visualized by UV radiation.

\section{Sensitivity and specificity of conventional PCR and the real-time fluorescence LAMP assay}

To prepare a plasmid DNA standard, the PCR product, amplified using the external primers of the $P$. jirovecii $18 \mathrm{~S}$ rRNA gene described above, was excised from the gel and purified using the agarose gel DNA extraction kit (Tiangen Biotech Co., LTD., Beijing, China). The recovered fragment was cloned into the TA cloning vector pMD18-T (Takara, Biotechnology Co., LTD., Dalian, China) with a standard TA cloning technique. DNA was extracted from one recombinant plasmid clone containing the correct sequence. DNA concentration was measured using the NanoDrop UV-Vis spectrophotometer (Thermo Fisher Scientific, USA). The sensitivities of the LAMP and PCR methods were compared using 10-fold serial dilutions of recombinant plasmid DNA (from $1 \times$ $10^{0}$ copies $/ \mu \mathrm{L}$ to $1 \times 10^{8}$ copies $/ \mu \mathrm{L}$ ) in triplicate.

The specificities of the real-time fluorescence LAMP assay and conventional PCR were determined using genomic DNA from 10 other common respiratory microorganisms, including Candida albicans, C. tropicalis, C. krusei, C. glabrata, C. parapsilosis, Aspergillus niger, Streptococcus pneumoniae, Escherichia coli, Acinetobacter baumannii, and Methicillin resistant Staphylococcus aureus (MRSA). The genomic DNA of each microorganism was extracted using the Plant Genomic DNA Kit (Tiangen Biotech Co., LTD., Beijing, China) or the TIANamp Bacteria DNA Kit (Tiangen Biotech Co., LTD., Beijing, China). An aliquot of 10-100 ng of genomic DNA from each microorganism was used in each reaction.

\section{Statistical analysis}

All data were analyzed using the statistical analysis software SPSS (version 20.0, IBM Corporation, Chicago, IL, USA). Counting variables are represented as frequencies or percentages. Continuous variables are represented as the mean \pm SD. Data related to clinical findings, imaging examinations, serologic parameters, and other indicators were compared using the $\chi^{2}$ test. Fisher's exact test was used for the clinical data analysis when the number of samples was less than 40 or the expected value was less than 1. $P$ values less than 0.05 were considered as statistically significant.

\section{Results}

Development of a modified real-time fluorescence LAMP assay for $P$. jirovecii detection

We developed a modified real-time fluorescence LAMP assay of $P$. jirovecii. This assay showed no cross-reactivity with 10 other common respiratory pathogenic microorganisms tested, suggesting its high specificity (Fig. 1). The sensitivity of this assay was compared with that of conventional PCR using serial dilutions of recombinant plasmid DNA containing P. jirovecii 18S rRNA gene. As shown in Figs. 2, the lowest detection threshold was $1 \times 10$ copies $/ \mu$ for the LAMP assay, which was 1000 times more sensitive than that of conventional PCR $\left(1 \times 10^{4}\right.$ copies $\left./ \mu \mathrm{l}\right)$.

We also compared the sensitivity of this modified LAMP assay with conventional PCR using clinical specimens of patients with various respiratory diseases. The positive rates of P. jirovecii were $69.7 \%(281 / 403)$ by the LAMP assay (Fig. 3) and $40.5 \%(163 / 403)$ by conventional PCR. In only one case, positive by both assays, cyst forms of $P$. jirovecii were detected by GMS and Giemsa staining. There was a good coincidence of the $P$. jirovecii detection rate between the LAMP and conventional PCR. All the positive specimens detected by conventional PCR were also positive in LAMP, however, the all positive specimens detected by LAMP were not necessarily positive in conventional PCR. All these data indicate that our modified real-time fluorescence LAMP method is reliable for the detection of $P$. jirovecii.

\section{Prevalence of $P$. jirovecii colonization in sputa and BALF from patients with various pulmonary diseases}

Of the 403 patients enrolled, the mean age was $66.7 \pm 12.5$ years, including 248 (61.5\%) males and 155 (38.5\%) females. The overall prevalence of $P$. jirovecii colonization amongst all enrolled patients was 69.7\% (281/403) (Table 1). The prevalence of $P$. jirovecii colonization in BALF of patients was 


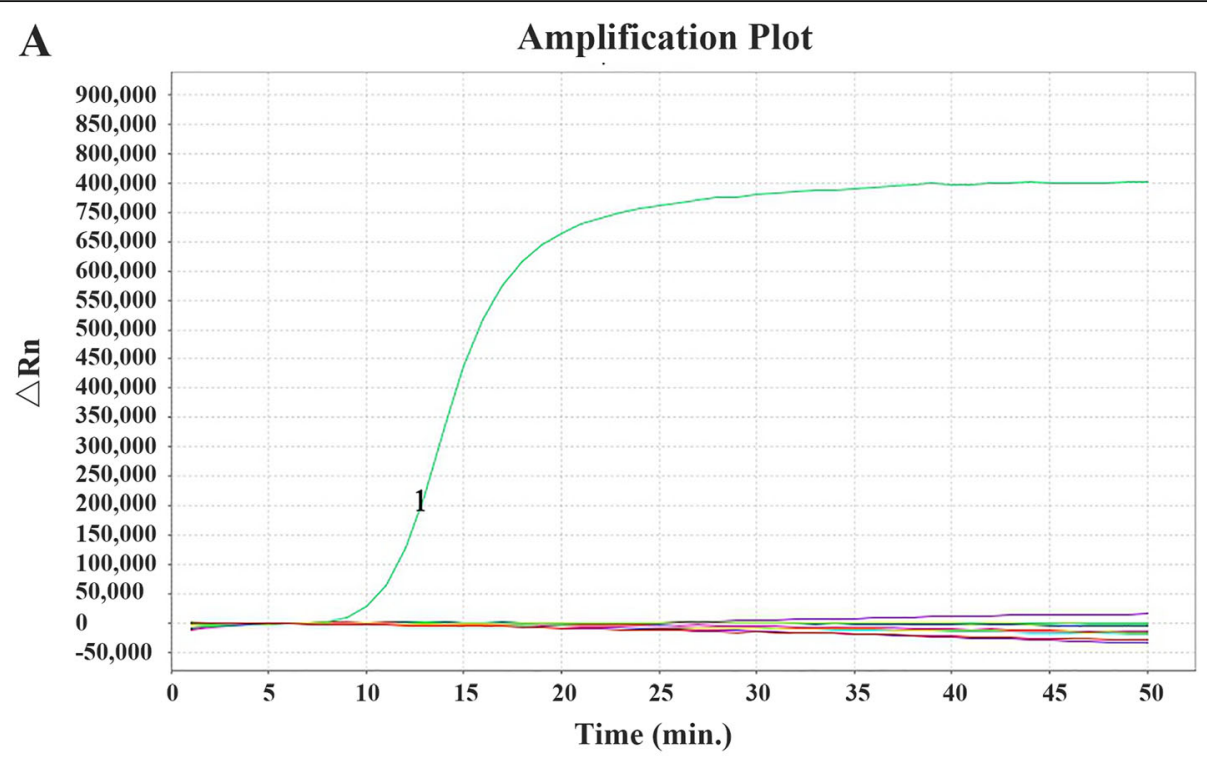

B

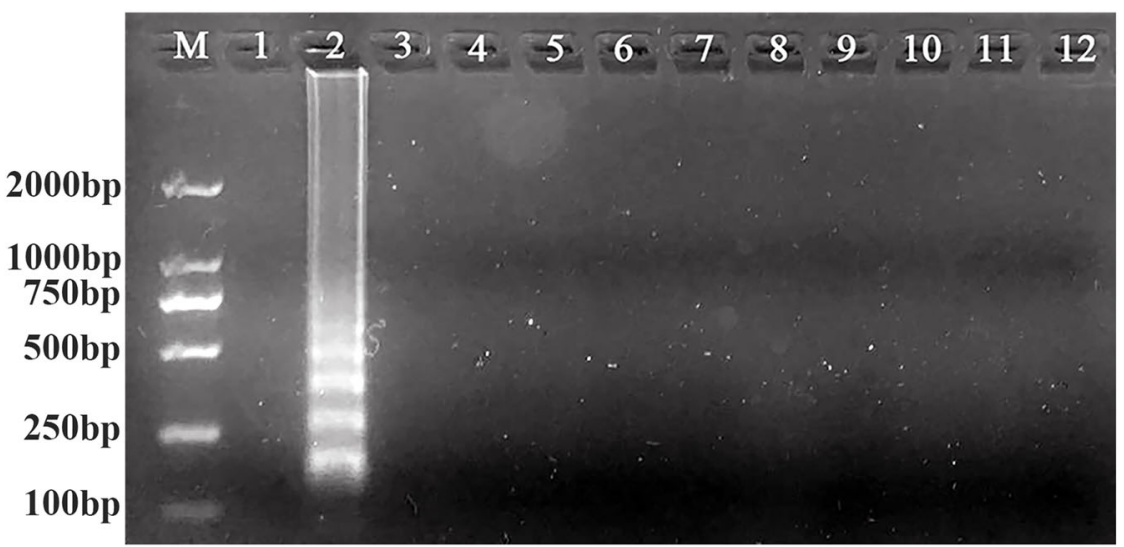

Fig. 1 The specificity of the real-time fluorescence LAMP assay for $P$. jirovecii detection. a A representative result of the LAMP assay. The green curve (labeled as 1) represents the LAMP amplification plot of one positive specimen of $P$. jirovecii. Other flat curves near the bottom represent amplification plots of control samples, including the negative control (with water) and other common respiratory microbes such as Candida albicans, C. tropicalis, C. krusei, C. glabrata, C. parapsilosis, Aspergillus niger, Streptococcus pneumoniae, Escherichia coli, Acinetobacter baumannii, and Methicillin resistant Staphylococcus aureus (MRSA). b A representative result of $2 \%$ agarose gel electrophoresis analysis of LAMP products. M, DNA size marker; lane 1, no template control; lane 2, P. jirovecii-positive specimen. Lanes 312 are C. albicans, C. tropicalis, C. krusei, C. glabrata, C. parapsilosis, A. nige, S. pneumoniae, E. coli, A. baumannii, and Methicillin resistant S. aureus (MRSA), respectively

higher compared with it in sputa, but the comparative differences between them were not significant $(75.9 \%$ vs $68.2 \%, P>0.05$ ) (Table 2). The prevalence of $P$. jirovecii colonization in ILDs patients was significantly higher compared with COPD and AECOPD patients (85\% vs 67 and $43.3 \%, P<0.01$ ). Furthermore, the prevalence of $P$. jirovecii colonization in AECOPD patients was significantly higher than those with stabilized COPD (67.4\% vs $43.3 \%$, $P<0.01$ ) (Table 1).

\section{Presence of various comorbidities in patients with $P$. jirovecii colonization and pulmonary diseases}

More than half ( 230 or $57 \%$ ) of all the enrolled patients presented with coexisting diseases including autoimmune diseases, connective tissue diseases, and other diseases (Table 3). Majority of these patients with comorbidities were positive for $P$. jirovecii (167 vs 63). There were 24 patients with autoimmune diseases including rheumatoid arthritis (RA), autoimmune thyroid disease (AITD), chronic glomerulonephritis (CGN), Sjogren's syndrome, connective tissue disease (CTD), and mixed connective tissue diseases (MCTD). Notably, the frequency of $P$. jirovecii detection in patients with autoimmune diseases was higher than those with the hypertension, diabetes, and coronary heart disease. Moreover, particular finding worth mentioning is the presence of a statistically significant difference in the frequency of $P$. jirovecii gene detection between patients with autoimmune diseases and those with the cardiac insufficiency $(P<0.05)$. 


\section{Amplification Plot}

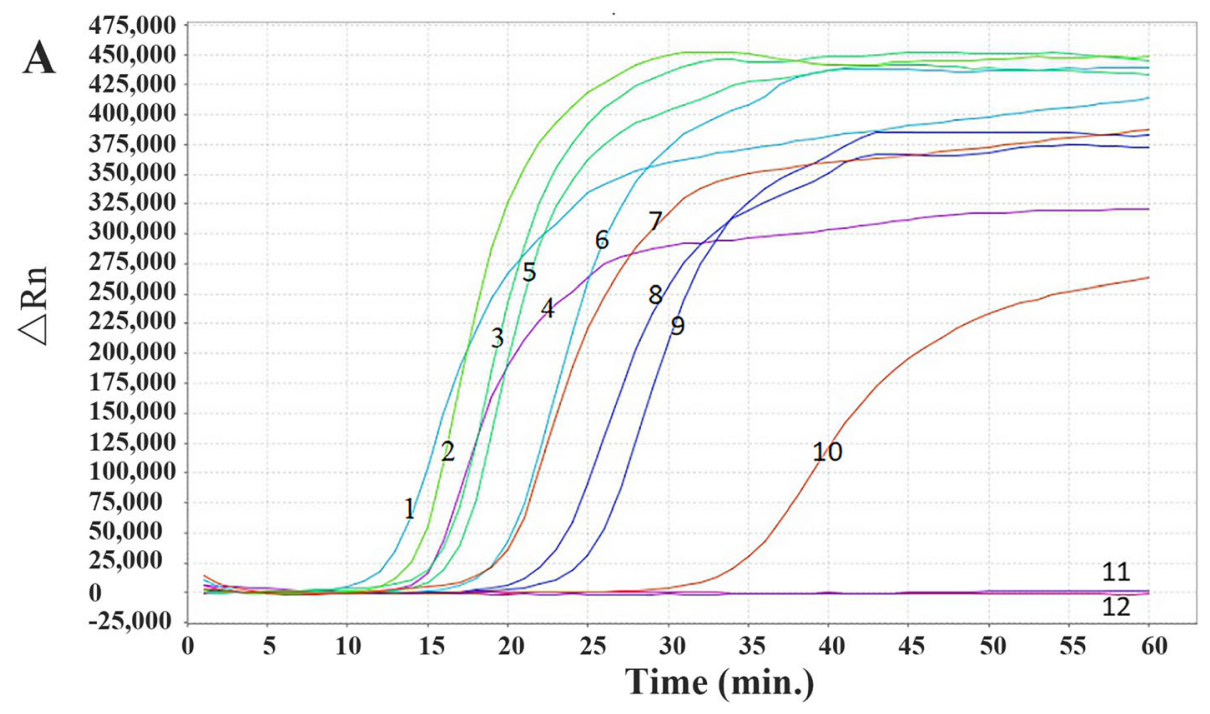

B

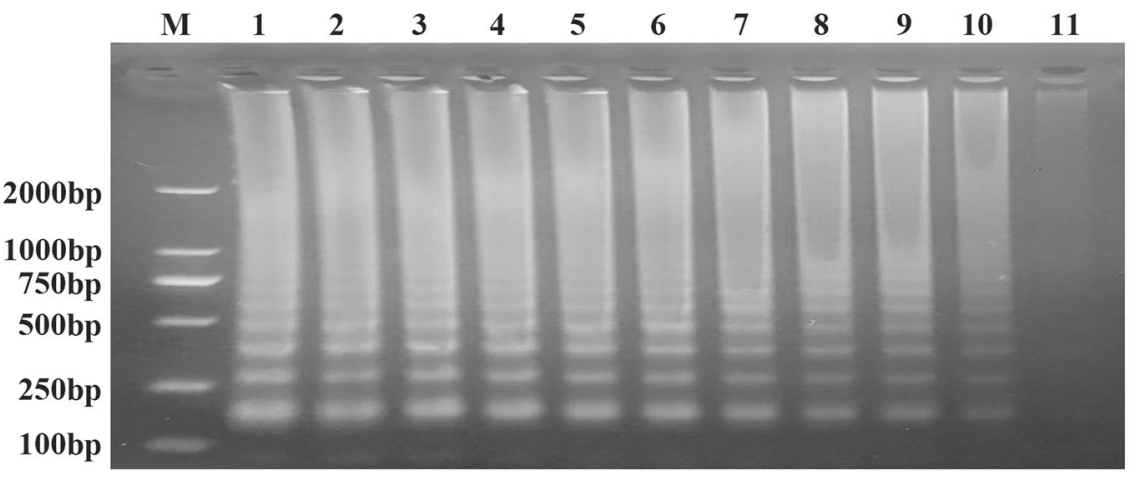

C

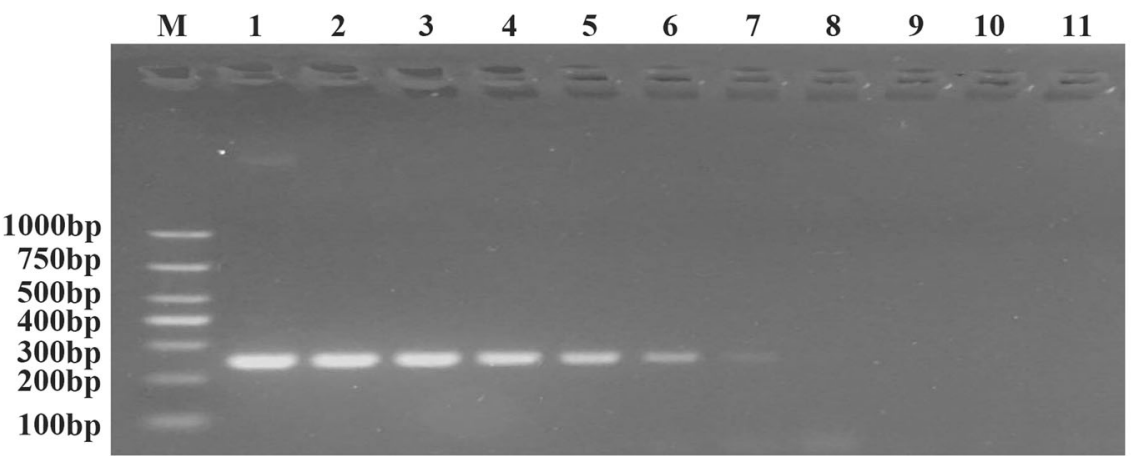

Fig. 2 Comparison of the sensitivity of real-time fluorescence LAMP assay and conventional PCR using 10-fold serial dilutions of recombinant plasmid DNA containing P. jirovecii $18 \mathrm{~S}$ rRNA gene. a Amplification plots of the real-time fluorescence LAMP assay (from $1 \times 10^{10} \mathrm{copies} / \mu \mathrm{l}$ for curve 1 to for $1 \times 10^{\circ}$ copies/ $\mu$ l curve 11, and no template control for curve 12). b 2\% agarose gel electrophoresis of LAMP products from serial dilutions of plasmid from $1 \times 10^{10}$ copies/ $\mu$ l for lane 1 to for $1 \times 10^{0}$ copies $/ \mu$ l lane 11 . M, DNA size marker. c $2 \%$ gel electrophoresis of conversional PCR products from serial dilutions of plasmid from $1 \times 10^{10}$ copies/ $\mu$ l for lane 1 to for $1 \times 10^{0}$ copies/ $\mu$ lane 11 . M, DNA size marker

\section{Concurrent infection with other fungi or bacteria in} patients with $P$. jirovecii colonization

Most of the patients with $P$. jirovecii colonization (71/ 102 or $69.6 \%$ ) had concurrent infection with other fungi and bacteria (Table 4). There were no significant differences in pair-wise comparison of the $P$. jirovecii colonization rates amongst patients co-infected with bacteria, those with other fungi, and those with both 


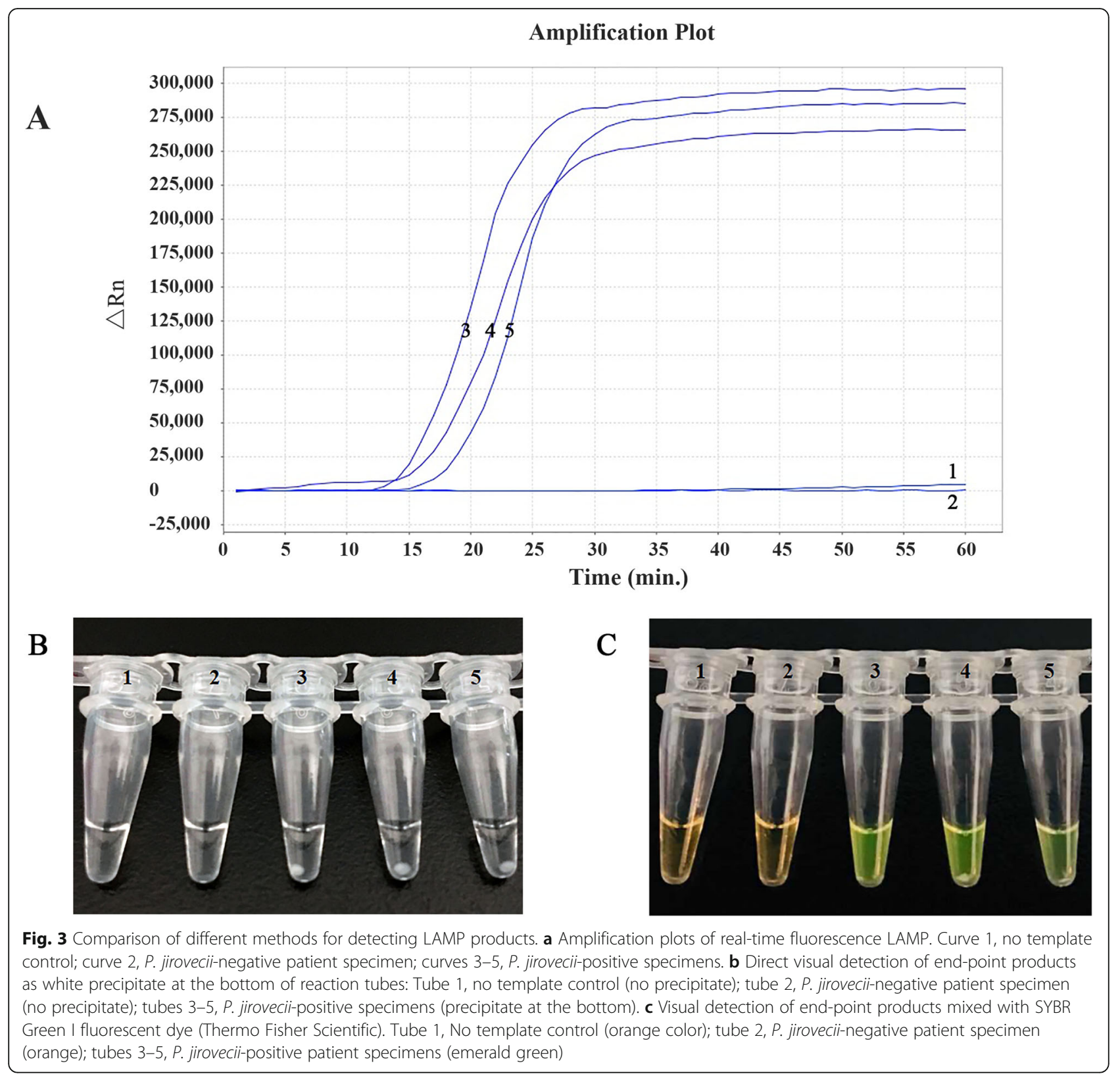

other fungi and bacteria $(66.2 \%$ vs $69.6 \%$ vs $85.7 \%$, $P>0.05)$. Furthermore, comparison between AECOPD patients co-infected with bacteria or fungi and COPD patients in stable stage co-infected with bacteria or fungi showed that there was no significant difference $(25.69 \%$ vs $33.33 \%, P>0.05$ ) (Table 5 ).

\section{Association of $P$. jirovecii colonization with clinical} characteristics of patients with various pulmonary diseases As illustrated in Table 6, no significant differences in PCT, LDH, and ESR measurements were observed between patients with and without $P$. jirovecii colonization $(P>0.05)$. However, patients with $P$. jirovecii colonization showed significantly increased levels of BDG and CRP, and significantly decreased levels of pulmonary function and CD4+ T-cell counts compared to patients without $P$. jirovecii colonization $(P<0.05)$. In addition, compared to patients without $P$. jirovecii colonization, patients with $P$. jirovecii colonization had significantly more abnormal findings on thoracic HRCT imaging, including reticulation opacities, cystic lesions, septal thickening, nodules, irregular linear opacities, and consolidation $(P<0.05)$.

\section{Discussion}

$P$. jirovecii is a life-threatening opportunistic pathogen in immunocompromised patients. As extensively reviewed by Morris and Norris, Pneumocystis colonization, in contrast to Pneumocystis infection, is defined as the 
Table $1 P$. jirovecii colonization rate in patients with pulmonary diseases

\begin{tabular}{|c|c|c|c|c|}
\hline & $\begin{array}{l}\text { Patients with colonization } \\
(n=281)\end{array}$ & $\begin{array}{l}\text { Patients without colonization } \\
(n=122)\end{array}$ & $\begin{array}{l}\text { All patients } \\
(n=403)\end{array}$ & Positive rate (\%) \\
\hline Age (years) & $66.21 \pm 13.23$ & $67.98 \pm 10.63$ & $66.74 \pm 12.51$ & \\
\hline Male (\%) & 66.5 & 33.5 & 61.5 & \\
\hline COPD & 160 & 88 & 248 & $64.52^{*}$ \\
\hline AECOPD & 147 & 71 & 218 & $67.43^{* *}$ \\
\hline Stable stage & 13 & 17 & 30 & 43.33 \\
\hline Interstitial lung diseases & 55 & 10 & 65 & 84.62 \\
\hline$\| P$ & 47 & 8 & 55 & 84.45 \\
\hline Sarcoidosis & 8 & 0 & 8 & 100 \\
\hline PAP & 0 & 2 & 2 & 0 \\
\hline AECB & 27 & 13 & 40 & 67.50 \\
\hline Bronchiectasis & 30 & 8 & 38 & 78.95 \\
\hline Bronchial asthma & 2 & 3 & 5 & 40.0 \\
\hline IPA & 4 & 0 & 4 & 100 \\
\hline Type 1 respiratory failure & 3 & 0 & 3 & 100 \\
\hline
\end{tabular}

Abbreviations: COPD chronic obstructive pulmonary disease, $A E C O P D$ acute exacerbation of COPD, $A E C B$ acute exacerbations of chronic bronchitis, IPA invasive pulmonary aspergillosis, PAP pulmonary alveolar proteinosis, IIP idiopathic interstitial pneumonia

Data are expressed as numbers (\%). ${ }^{*} P<0.01$ compared with ILDs; $P>0.05$ compared with AECB, bronchiectasis and bronchial asthma; ${ }^{* *} P<0.01$ compared with the stable stage

presence of Pneumocystis organisms (usually identified by PCR-based molecular biological techniques) without signs or symptoms of acute pneumonia $[2,8]$. Colonization with $P$. jirovecii can even occur in individuals with normal immunity due to the development of highly efficient strategies to escape both innate and acquired immune defenses in host $[1,2]$. These colonized individuals may serve as reservoirs of nosocomial infections. A high-level prevalence of $P$. jirovecii colonization in immunocompetent individuals has been reported in numerous studies throughout the world [2,29]. The role of $P$. jirovecii colonization in certain pulmonary diseases, particularly amongst patients with COPD, has gained increasing attention $[5,8,9,30]$. In the present multicenter study involving 403 non-HIV patients with various pulmonary diseases, we found a high prevalence of $P$. jirovecii colonization and this was associated with some clinical and laboratory characteristics of patients with pulmonary diseases, supporting the involvement of $P$. jirovecii colonization in pulmonary diseases.

A similar study, LAMP assays for the detection of $P$. jirovecii gene amongst the patients with chronic pulmonary diseases, was previously performed in our hospital in 2015 [5].
SYBR Green I, a fluorescent dye, had been used previously in this assay. However, SYBR Green I, used as the conventional intercalating fluorescent dye, had several drawbacks, including significant inhibition, despite its widespread use for LAMP reaction and real-time PCR [17, 18]. SYTO 9.0 has been confirmed as having the least inhibitory effect amongst the fluorescent intercalating dyes tested [17]. In order to avoid inhibition of the LAMP reaction due to SYBR Green I, we first developed a modified real-time fluorescence LAMP assay by using SYTO 9.0 (Thermo Fisher Scientific), as the fluorescent dye for P. jirovecii. Compared with the conventional PCR, the modified LAMP assay showed 1000 times more sensitivity, whereas the LAMP assay reported previously was 200 times more sensitive. We found that the lowest detection threshold for this modified assay was lower than that reported in the previous study using indirect comparison (10 copies vs 50 copies per reaction), which indicated that the sensitivity of detection has been modified. So, in this study, SYTO 9.0 fluorescent dye, added to the reaction of modified real-time fluorescence LAMP assay mixture, demonstrated higher amplification efficiency and sensitivity, without detectable inhibitory effect [17]. To better assess the prevalence of $P$. jirovecii colonization, we used this modified

Table 2 P. jirovecii colonization rate in BALF and sputa of the patients

\begin{tabular}{lllll}
\hline & $\begin{array}{l}\text { Patients with colonization } \\
(n=281)\end{array}$ & $\begin{array}{l}\text { Patients without colonization } \\
(n=122)\end{array}$ & $\begin{array}{l}\text { Total } \\
(n=403)\end{array}$ & Positive rate (\%) \\
\hline P. jirovecii colonization in BALF & 60 & 19 & 79 & $75.94^{*}$ \\
P. jirovecii colonization in sputa & 221 & 103 & 324 & 68.20 \\
\hline
\end{tabular}

${ }^{*} P>0.05$ compared with $P$. jirovecii colonization in sputa 
Table 3 Comorbidities in patients with P. jirovecii colonization

\begin{tabular}{lllll}
\hline Comorbidities & Patients with colonization & Patients without colonization & Total & Positive rate (\%) \\
\hline Hypertension & 45 & 12 & 57 & 78.95 \\
Cardiac insufficiency & 31 & 20 & 51 & 60.78 \\
Diabetes mellitus & 25 & 8 & 33 & 75.76 \\
Coronary heart disease & 24 & 9 & 33 & 72.73 \\
Autoimmune diseases & 24 & 5 & 29 & $82.75^{\#}$ \\
Systemic inflammatory response syndrome & 9 & 2 & 11 & 81.82 \\
Others & 9 & 7 & 16 & 56.25 \\
Total & 167 & 63 & 230 & 72.61 \\
\hline
\end{tabular}

${ }^{\#} P>0.05$ compared with hypertension, diabetes, coronary heart disease and systemic inflammatory response syndrome; $P<0.05,>0.01$, compared with the cardiac insufficiency group

real-time fluorescence LAMP assay for the detection of $P$. jirovecii. The overall frequency of colonization by $P$. jirovecii (69.7\%), observed in this study, appears to be higher than that reported in previous studies $(16-63.3 \%)$ [5, 31]. Moreover, in this study, the prevalence of $P$. jirovecii colonization in BALF of patients (75.9\%) is higher than that reported previously (21/30 or $70 \%$ ) [30]. In addition, Nested PCR (mt LSU rRNA as Pneumocystis target gene) was compared with real-time fluorescence LAMP, and the positive rate was $58.56 \%$ (236/403), which was lower than the positive rate of real-time fluorescence LAMP used in this study. The reasons for these observed differences are unclear. It may be related to the superiorities or advantages of this modified LAMP mentioned above, in addition, also related to different target genes, different detection methods, different sample processing, different underlying diseases and diagnosis, or different patient population tested.

The high prevalence of $P$. jirovecii colonization observed in this study is consistent with elevated serum beta-glucan levels in patients with $P$. jirovecii colonization compared to patients without $P$. jirovecii colonization (Table 6), as has been reported in various studies [32-34]. In addition to the high prevalence of $P$. jirovecii colonization in various pulmonary diseases, this study provides several other lines of evidence supporting $P$. jirovecii colonization as a risk factor for the development of pulmonary diseases. In this study, HRCT imaging findings suggested that the ground-glass opacity (GGO) could be a result of $P$. jirovecii colonization in patients with pulmonary diseases. Compared to patients without $P$. jirovecii colonization, patients with $P$. jirovecii colonization showed a decreased pulmonary function as assessed by the FEV1/FVC values (Table 6), which may suggest a possibility of damage to the respiratory tract due to long-term colonization. This possibility is further supported by the elevated serum C-reactive protein (CRP) levels in patients with $P$. jirovecii colonization, as CRP is a sensitive marker

Table 4 Concurrent infection with other fungi and bacteria in patients with $P$. jirovecii colonization

\begin{tabular}{lllll}
\hline Microorganism & Positive & Negative & Total & Positive rate (\%) \\
\hline Bacteria & 43 & 22 & 65 & $66.15^{*}$ \\
Streptococcus pneumoniae & 10 & 5 & 15 & 66.67 \\
Pseudomonas aeruginosa & 11 & 3 & 14 & 78.57 \\
Klebsiella pneumoniae & 5 & 4 & 9 & 55.56 \\
Haemophilus influenzae & 4 & 3 & 5 & 57.14 \\
Acinetobacter baumannii & 4 & 1 & 15 & 80.00 \\
Others & 9 & 6 & 23 & 60.00 \\
Other fungi & 16 & 7 & 17 & 69.57 \\
Candida albicans & 11 & 6 & 4 & 64.71 \\
Aspergillus fumigatus & 4 & 0 & 2 & 100 \\
Others & 1 & 1 & 14 & 50.00 \\
Other fungi and bacteria & 12 & 2 & 3 & 85.71 \\
P. aeruginosa and C. albicans & 3 & 0 & 11 & 100 \\
Others & 9 & 2 & 102 & 81.82 \\
Total & 71 & 31 & & 69.61 \\
\hline
\end{tabular}

${ }^{*} P>0.05$ compared with fungal infection alone and infection with both fungi bacteria 
Table 5 Concurrent infection with other fungi or bacteria in patients with COPD

\begin{tabular}{lllll}
\hline & Patients with concurrent infection & Patients without concurrent infection & Total & Positive rate (\%) \\
\hline AECOPD & 56 & 162 & 218 & $25.69^{*}$ \\
COPD Stable stage & 10 & 20 & 30 & 33.33 \\
Total & 66 & 182 & 248 & 36.26 \\
\hline
\end{tabular}

${ }^{\bar{*}} \mathrm{P}>0.05$ compared with concurrent infection with fungi or bacteria among COPD patients in stable stage

of inflammation and tissue damage. Finally, consistent with the previous studies [35-37], we also observed decreased CD4+ T-cell counts in patients with P. jirovecii colonization compared to patients without $P$. jirovecii colonization, which suggests an impaired $\mathrm{T}$ cell-mediated immunity.
Patients with chronic pulmonary diseases frequently suffer from various comorbidities (Table 3 ), which may also contribute to the severity of diseases. In this study, we found a high rate of underlying autoimmune diseases in patients with $P$. jirovecii colonization (Table 3). This finding can be explained by the widespread

Table 6 Association of P. jirovecii colonization with laboratory findings of the patients

\begin{tabular}{|c|c|c|c|c|}
\hline & Positive & Negative & Total & Positive rate (\%) \\
\hline \multicolumn{5}{|l|}{ Serum BDG levels } \\
\hline Positive (> $10 \mathrm{pg} / \mathrm{ml}$ ) & 34 & 5 & 39 & $81.18^{a}$ \\
\hline Negative $(<10 \mathrm{pg} / \mathrm{ml})$ & 128 & 50 & 178 & 71.91 \\
\hline \multicolumn{5}{|l|}{ Serum PCT levels } \\
\hline Positive (> $0.5 \mathrm{ng} / \mathrm{mL}$ ) & 59 & 22 & 81 & $72.84^{\mathrm{b}}$ \\
\hline Negative $(<0.5 \mathrm{ng} / \mathrm{mL})$ & 103 & 33 & 136 & 75.74 \\
\hline \multicolumn{5}{|l|}{ Serum LDH levels } \\
\hline Positive (> 225 U/L) & 84 & 33 & 117 & $71.79^{c}$ \\
\hline Negative (<225 U/L) & 197 & 89 & 286 & 68.88 \\
\hline \multicolumn{5}{|l|}{ Serum CRP levels } \\
\hline Positive (> 5 mg/L) & 171 & 59 & 230 & $74.35^{d}$ \\
\hline Negative (<5 mg/L) & 110 & 63 & 173 & 63.58 \\
\hline \multicolumn{5}{|l|}{ ESR test } \\
\hline Abnormal & 78 & 33 & 111 & $70.27^{e}$ \\
\hline Normal & 109 & 51 & 160 & 68.13 \\
\hline \multicolumn{5}{|l|}{ Thoracic HRCT findings } \\
\hline GGO positive & 67 & 14 & 81 & $82.72^{f}$ \\
\hline GGO negative & 95 & 41 & 136 & 69.85 \\
\hline \multicolumn{5}{|l|}{ Pulmonary function } \\
\hline FEV1/FVC positive $(<70 \%)$ & 25 & 6 & 31 & $80.65^{9}$ \\
\hline FEV1/FVC negative (> 70\%) & 14 & 11 & 25 & 56.00 \\
\hline \multicolumn{5}{|l|}{$\mathrm{CD}_{4}^{+} \mathrm{T}$-cell count } \\
\hline$<410 / \mathrm{mm}^{3}$ & 23 & 2 & 25 & $92.00^{h}$ \\
\hline$>410 / \mathrm{mm}^{3}$ & 53 & 24 & 77 & 68.83 \\
\hline
\end{tabular}

Abbreviations: $B D G$ 1,3- $\beta$-D-glucan, $P C T$ pro-calcitonin, $L D H$ lactate dehydrogenase, $C R P$ C-reactive protein, ESR erythrocyte sedimentation rate, GGO ground-glass opacity, HRCT high-resolution computed tomography, FEV1 forced expiratory volume in one second, FVC forced vital capacity

${ }^{\mathrm{a}} P<0.05,>0.01$, Compared with serum BDG $<10 \mathrm{pg} / \mathrm{mL}$

${ }^{\mathrm{b}} P>0.05$ Compared with serum $\mathrm{PCT}<0.5 \mathrm{ng} / \mathrm{mL}$

${ }^{c} P>0.05$ compared with LDH levels $<225 \mathrm{U} / \mathrm{L}$

${ }^{d} P<0.05,>0.01$, compared with CRP negative $(<5 \mathrm{mg} / \mathrm{L})$

${ }^{\mathrm{e}} P>0.05$ compared with ESR normal. ESR abnormal $(>15 \mathrm{~mm} / \mathrm{h}$ for men and $>20 \mathrm{~mm} / \mathrm{h}$ for women). ESR normal $(\leq 15 \mathrm{~mm} / \mathrm{h}$ for men and $\leq 20 \mathrm{~mm} / \mathrm{h}$ for women)

${ }^{\mathrm{f}} P<0.05,>0.01$, compared with GGO negative

${ }^{9} P<0.05,>0.01$, compared with FEV1/FVC negative $(>70 \%)$

${ }^{\mathrm{h}} P<0.05,>0.01$, compared with CD4+ T-cell count $>410 / \mathrm{mm}^{3}$ 
administration of systemic high-dose steroids in these patients [38, 39].

Despite increasing reports of $P$. jirovecii colonization, there are only a few studies of coinfection with other respiratory pathogens in patients with $P$. jirovecii colonization [40-42]. To study whether the presence of other microorganisms in lungs facilitate or inhibit the $P$. jirovecii colonization, the concurrent infection with other fungi and bacteria in patients with $P$. jirovecii colonization were investigated. And, we found that the majority of patients with $P$. jirovecii colonization had concurrent infections with other fungal or bacterial pathogens (Table 4). Although the presence of other organisms might be the most common reason for the association with exacerbation in COPD, in this study, there was no significant difference in comparison of the prevalence of concurrent infection with other organisms in AECOPD patients and those with stabilized COPD (Table 5). In addition, PCT is a sensitive biomarker of bacterial infection. However, no significant differences in PCT measurements were observed between patients with and without $P$. jirovecii colonization. This indirectly indicates that $P$. jirovecii colonization might contribute to the risk of COPD exacerbations and other various pulmonary diseases. Further studies are needed to assess the differential contribution of these pathogens to the pulmonary diseases.

This study did have certain limitations. First, a direct comparison between the LAMP assay previously reported and the modified assay established in this study had not been performed yet. Further analysis should be performed after the direct comparison of the assays in the future clinical studies. Second, there were certain heterogeneities in the sample types (sputum and BALF samples) and underlying clinical conditions, which might have caused biases in data interpretation. Third, we did not subtract coinfection with other fungi or bacteria when assessing the association of $P$. jirovecii colonization with clinical characteristics due to the small sample size after subtraction. Fourth, sputum specimens are not the best specimens to examine the prevalence of coinfection with other fungi or bacteria in the lungs, due to unavoidable contamination with normal microbial flora in the oral cavity and nasopharynx. In this study, we used a large number of sputum specimens, due to the limited availability of BALF samples. Lastly, we represented the data on the basis of individual pulmonary diseases; however, due to retrospective nature of this study, we lacked the data on severity of individual pulmonary disease and thus, could not represent the data on the basis of severity, such as the GOLD staging for COPD and the severity classification of asthma.

\section{Conclusions}

In summary, we have developed a modified LAMP assay for detecting $P$. jirovecii colonization. This multi-center study involving 403 patients, using this assay, has provided additional new evidence for the involvement of $P$. jirovecii colonization in the development of pulmonary diseases in non-HIV patients, and highlights the need to further study the pathogenesis and transmission of $P$. jirovecii in pulmonary diseases.

\begin{abstract}
Abbreviations
PJP: Pneumocystis jirovecii pneumonia; COPD: Chronic obstructive pulmonary disease; HIV: Human immunodeficiency virus; LAMP: Loop-mediated isothermal amplification; AECOPD: Acute exacerbations of chronic obstructive pulmonary disease; AECB: Acute exacerbations of chronic bronchitis; ILDs: Interstitial lung diseases; IPA: Invasive pulmonary aspergillosis; BALF: Bronchoalveolar lavage fluid; ESR: Erythrocyte sedimentation rate; LDH: Lactate dehydrogenase; PCT: Pro-calcitonin; CRP: Creactive protein; BDG: 1,3-ß-D-glucan; HRCT: High-resolution computed tomography; GGO: The ground-glass opacity
\end{abstract}

\section{Acknowledgements}

We would like to thank for the assistance from many healthcare workers involved in this study from the following units including the Department of Respiratory Internal Medicine of the First Affiliated Hospital of China Medical University, the Department of Respiratory Internal Medicine and Clinical Laboratory of the Fourth Affiliated Hospital of China Medical University, the Department of Respiratory Internal Medicine of the Fifth Affiliated Hospital of China Medical University, the Department of Respiratory Internal Medicine and the Clinical Laboratory of the Eleventh Affiliated Hospital of Shanxi Medical University and the Department of Respiratory Internal Medicine of the General Hospital of Shenyang Military Command.

\section{Authors' contributions}

This study was designed and conceived by $\mathrm{XT}$ and $\mathrm{AC}$, and performed and analyzed by XT. Data acquisition, statistical analysis and manuscript drafting were performed by $X T$. MZ, LF, DW, and HL participated in the specimen collection and patients' management. WJ and AC reviewed and edited the manuscript. All authors contributed to revising the paper and analyzing data. All authors have read and approved the final manuscript and are responsible for all aspects of this study.

\section{Funding}

This study was supported by Natural Science Foundation of China (Project81370189). The funding agency had no role in the design of the study, the collection, analysis and interpretation of the data and the preparation of the manuscript.

\section{Availability of data and materials}

The data used in this study are available from the corresponding author on reasonable request.

\section{Ethics approval and consent to participate}

This retrospective study was approved by the Institutional Ethics Review Board, China Medical University (2013070). Written informed consent was obtained from all the patients involved in this study.

\section{Consent for publication}

Not applicable.

\section{Competing interests}

The authors declared that they have no competing interests.

\section{Author details}

'Department of Microbiology and Parasitology, College of Basic Medical Science, China Medical University, 77 Puhe Road, Shenyang, People's Republic of China. ${ }^{2}$ Department of Respiratory and Critical Care Medicine, First Hospital of Shanxi Medical University, Taiyuan, People's Republic of China. ${ }^{3}$ Department of Respiratory Medicine, General Hospital of Shenyang 
Military Command, Shenyang, People's Republic of China. ${ }^{4}$ Department of Respiratory Internal Medicine, the First Affiliated Hospital of China Medical University, Shenyang, People's Republic of China. ${ }^{5}$ Department of Medical Laboratory, the Eleventh Affiliated Hospital of Shanxi Medical University, Lvliang, People's Republic of China. ${ }^{6}$ Department of Immunology, College of Basic Medical Science, China Medical University, Shenyang, People's Republic of China.

Received: 4 June 2019 Accepted: 12 March 2020

Published online: 20 March 2020

\section{References}

1. Ma L, Chen Z, Huang da W, Kutty G, Ishihara M, Wang H, Abouelleil A Bishop L, Davey E, Deng R, et al. Genome analysis of three Pneumocystis species reveals adaptation mechanisms to life exclusively in mammalian hosts. Nat Commun. 2016;7:10740.

2. Ma L, Cisse $\mathrm{OH}$, Kovacs JA. A molecular window into the biology and epidemiology of Pneumocystis spp. Clin Microbiol Rev. 2018;31(3):1-49.

3. Asai N, Aoshima M, Ohkuni $Y$, Kobayashi H, Matsunuma R, Nakashima K, Katsurada N, Nakano H, Otsuka Y, Kawamura Y, et al. A successful diagnostic case of Pneumocystis pneumonia by the loop-mediated isothermal amplification method in a patient with dermatomyositis. J Infect Chemother. 2012;18(6):965-9.

4. Omer O, Cohen P, Neong SF, Smith GV. Pneumocystis pneumonia complicating immunosuppressive therapy in Crohns disease: a preventable problem? Frontline Gastroenterol. 2016;7(3):222-6.

5. Wang DD, Zheng MQ, Zhang N, An CL. Investigation of Pneumocystis jirovecii colonization in patients with chronic pulmonary diseases in the People's Republic of China. Int J Chron Obstruct Pulmon Dis. 2015;10: 2079-85.

6. Wieruszewski PM, Barreto JN, Frazee E, Daniels CE, Tosh PK, Dierkhising RA, Mara KC, Limper AH. Early corticosteroids for Pneumocystis pneumonia in adults without HIV are not associated with better outcome. Chest. 2018; 154(3):636-44.

7. Ye WL, Tang N, Wen YB, Li H, Li MX, Du B, Li XM. Underlying renal insufficiency: the pivotal risk factor for Pneumocystis jirovecii pneumonia in immunosuppressed patients with non-transplant glomerular disease. Int Urol Nephrol. 2016;48(11):1863-71.

8. Morris A, Norris KA. Colonization by Pneumocystis jirovecii and its role in disease. Clin Microbiol Rev. 2012;25(2):297-317.

9. Khalife S, Aliouat EM, Aliouat-Denis CM, Gantois N, Devos P, Mallat H, DeiCas E, Dabboussi F, Hamze M, Frealle E. First data on Pneumocystis jirovecii colonization in patients with respiratory diseases in North Lebanon. New Microbes New Infect. 2015;6:11-4.

10. Ozkoc S, Bayram Delibas S. Investigation of Pneumocystis jirovecii pneumonia and colonization in iatrogenically immunosuppressed and immunocompetent patients. Mikrobiyol Bul. 2015;49(2):221-30.

11. Vera C, Aguilar YA, Velez LA, Rueda ZV. High transient colonization by Pneumocystis jirovecii between mothers and newborn. Eur J Pediatr. 2017; 176(12):1619-27.

12. Danesi $P$, Ravagnan $S$, Johnson LR, Furlanello T, Milani A, Martin P, Boyd $S$, Best M, Galgut B, Irwin P, et al. Molecular diagnosis of Pneumocystis pneumonia in dogs. Med Mycol. 2017;55(8):828-42.

13. Fauchier T, Hasseine L, Gari-Toussaint M, Casanova V, Marty PM, Pomares C. Detection of Pneumocystis jirovecii by quantitative PCR to differentiate colonization and pneumonia in Immunocompromised HIV-positive and HIVnegative patients. J Clin Microbiol. 2016;54(6):1487-95.

14. Khodadadi $H$, Mirhendi H, Mohebali M, Kordbacheh $\mathrm{P}$, Zarrinfar H, Makimura K. Pneumocystis jirovecii colonization in non-HIV-infected patients based on nested-PCR detection in Bronchoalveolar lavage samples. Iran J Public Health. 2013;42(3):298-305.

15. Maillet M, Maubon D, Brion JP, Francois P, Molina L, Stahl JP, Epaulard O, Bosseray A, Pavese P. Pneumocystis jirovecii (Pj) quantitative PCR to differentiate Pj pneumonia from Pj colonization in immunocompromised patients. Eur J Clin Microbiol Infect Dis. 2014;33(3):331-6.

16. Notomi T, Okayama H, Masubuchi H, Yonekawa T, Watanabe K, Amino N, Hase T. Loop-mediated isothermal amplification of DNA. Nucleic Acids Res. 2000;28(12):E63.

17. Oscorbin IP, Belousova EA, Zakabunin Al, Boyarskikh UA, Filipenko ML. Comparison of fluorescent intercalating dyes for quantitative loop-mediated isothermal amplification (qLAMP). BioTechniques. 2016;61(1):20-5.
18. Eischeid AC. SYTO dyes and EvaGreen outperform SYBR green in real-time PCR. BMC Res Notes. 2011;4:263.

19. Medrano FJ, Montes-Cano M, Conde M, de la Horra C, Respaldiza N, Gasch A, Perez-Lozano MJ, Varela JM, Calderon EJ. Pneumocystis jirovecii in general population. Emerg Infect Dis. 2005;11(2):245-50.

20. Vogelmeier CF, Criner GJ, Martinez FJ, Anzueto A, Barnes PJ, Bourbeau J, Celli BR, Chen R, Decramer M, Fabbri LM, et al. Global strategy for the diagnosis, management, and prevention of chronic obstructive lung disease 2017 report: GOLD executive summary. Arch Bronconeumol. 2017;53(3):12849.

21. Qaseem A, Wilt TJ, Weinberger SE, Hanania NA, Criner G, van der Molen T, Marciniuk DD, Denberg T, Schunemann H, Wedzicha W, et al. Diagnosis and management of stable chronic obstructive pulmonary disease: a clinical practice guideline update from the American College of Physicians, American College of Chest Physicians, American Thoracic Society, and European Respiratory Society. Ann Intern Med. 2011;155(3):179-91.

22. Cottin V, Hirani NA, Hotchkin DL, Nambiar AM, Ogura T, Otaola M, Skowasch D, Park JS, Poonyagariyagorn HK, Wuyts W, et al. Presentation, diagnosis and clinical course of the spectrum of progressive-fibrosing interstitial lung diseases. Eur Respir Rev. 2018;27(150):1-11.

23. Raghu G, Collard HR, Egan JJ, Martinez FJ, Behr J, Brown KK, Colby TV, Cordier JF, Flaherty KR, Lasky JA, et al. An official ATS/ERS/JRS/ALAT statement: idiopathic pulmonary fibrosis: evidence-based guidelines for diagnosis and management. Am J Respir Crit Care Med. 2011;183(6):788824.

24. Nicolau DP, Sutherland C, Winget D, Baughman RP. Bronchopulmonary pharmacokinetic and pharmacodynamic profiles of levofloxacin $750 \mathrm{mg}$ once daily in adults undergoing treatment for acute exacerbation of chronic bronchitis. Pulm Pharmacol Ther. 2012;25(1):94-8.

25. Dodd JD, Lavelle LP, Fabre A, Brady D. Imaging in cystic fibrosis and noncystic fibrosis bronchiectasis. Semin Respir Crit Care Med. 2015;36(2):194206.

26. Smith MP. Diagnosis and management of bronchiectasis. CMAJ. 2017; 189(24):E828-35.

27. Wasche A, Schreiber J. Pathophysiology, diagnosis and treatment of asthma: focusing on a chronic disease. Anasthesiol Intensivmed Notfallmed Schmerzther. 2016;51(6):392-9.

28. Vo P, Kharasch VS. Respiratory failure. Pediatr Rev. 2014;35(11):476-84 quiz 485-476.

29. Rojas P, Friaza V, Garcia E, de la Horra C, Vargas SL, Calderon EJ, Pavon A. Early acquisition of Pneumocystis jirovecii colonization and potential association with respiratory distress syndrome in preterm newborn infants. Clin Infect Dis. 2017;65(6):976-81.

30. Ozkoc S, Bayram Delibas S, Erbaycu AE, Erguden C, Akisu C. Investigation of Pneumocystis jirovecii colonization in patients with pulmonary diseases. Turkiye Parazitol Derg. 2014;38(4):214-9.

31. Mekinian A, Durand-Joly I, Hatron PY, Moranne O, Denis G, Dei-Cas E, Morell-Dubois S, Lambert M, Launay D, Delhaes L, et al. Pneumocystis jirovecii colonization in patients with systemic autoimmune diseases: prevalence, risk factors of colonization and outcome. Rheumatology. 2011; 50(3):569-77.

32. Matsumura $Y$, Ito $Y$, linuma $Y$, Yasuma $K$, Yamamoto $M$, Matsushima $A$, Nagao M, Takakura S, Ichiyama S. Quantitative real-time PCR and the (1->3)-beta-D-glucan assay for differentiation between Pneumocystis jirovecii pneumonia and colonization. Clin Microbiol Infect. 2012;18(6):591-7.

33. Onishi A, Sugiyama D, Kogata Y, Saegusa J, Sugimoto T, Kawano $S$, Morinobu A, Nishimura K, Kumagai S. Diagnostic accuracy of serum 1,3beta-D-glucan for pneumocystis jiroveci pneumonia, invasive candidiasis, and invasive aspergillosis: systematic review and meta-analysis. J Clin Microbiol. 2012;50(1):7-15

34. Tasaka S, Kobayashi S, Yagi K, Asami T, Namkoong H, Yamasawa W, Ishii M, Hasegawa N, Betsuyaku T. Serum (1 --> 3) beta-D-glucan assay for discrimination between Pneumocystis jirovecii pneumonia and colonization. J Infect Chemother. 2014;20(11):678-81.

35. Kling HM, Shipley TW, Patil S, Morris A, Norris KA. Pneumocystis colonization in immunocompetent and simian immunodeficiency virus-infected cynomolgus macaques. J Infect Dis. 2009;199(1):89-96.

36. Mansharamani NG, Balachandran D, Vernovsky I, Garland R, Koziel H. Peripheral blood CD4 + T-lymphocyte counts during Pneumocystis carinii pneumonia in immunocompromised patients without HIV infection. Chest. 2000;118(3):712-20. 
37. Overgaard UM, Helweg-Larsen J. Pneumocystis jiroveci pneumonia (PCP) in HIV-1-negative patients: a retrospective study 2002-2004. Scand J Infect Dis. 2007;39(6-7):589-95.

38. Chew LC, Maceda-Galang LM, Tan YK, Chakraborty B, Thumboo J.

Pneumocystis jirovecii pneumonia in patients with autoimmune disease on high-dose glucocorticoid. J Clin Rheumatol. 2015;21(2):72-5.

39. Park JW, Curtis JR, Moon J, Song YW, Kim S, Lee EB. Prophylactic effect of trimethoprim-sulfamethoxazole for pneumocystis pneumonia in patients with rheumatic diseases exposed to prolonged high-dose glucocorticoids. Ann Rheum Dis. 2018;77(5):644-9.

40. Korkmaz Ekren P, Toreyin ZN, Nahid P, Doskaya M, Caner A, Turgay N, Zeytinoglu A, Toz S, Bacakoglu F, Guruz Y, et al. The association between Cytomegalovirus co-infection with Pneumocystis pneumonia and mortality in immunocompromised non-HIV patients. Clin Respir J. 2018;12(11):2590-7.

41. Mamoudou S, Bellaud G, Ana C, Gilles P. Lung co-infection by Pneumocystis jirovecii and Pseudomonas aeruginosa in AIDS: report of two cases. Pan Afr Med J. 2015;21:95.

42. Markantonatou AM, loakimidou A, Arvaniti K, Manou E, Papadopoulos V, Kiriklidou P, Samaras K, Kioumi A, Vyzantiadis TA. Pulmonary co-infections by Pneumocystis jirovecii and Aspergillus fumigatus in non-HIV patients: a report of two cases and literature review. Mycoses. 2017;60(10):626-33.

\section{Publisher's Note}

Springer Nature remains neutral with regard to jurisdictional claims in published maps and institutional affiliations.

Ready to submit your research? Choose BMC and benefit from:

- fast, convenient online submission

- thorough peer review by experienced researchers in your field

- rapid publication on acceptance

- support for research data, including large and complex data types

- gold Open Access which fosters wider collaboration and increased citations

- maximum visibility for your research: over $100 \mathrm{M}$ website views per year

At BMC, research is always in progress.

Learn more biomedcentral.com/submissions 